\title{
Snoring and the role of the GDP: British Society of Dental Sleep Medicine (BSDSM) pre-treatment screening protocol
}

- Suggests a protocol which will facilitate screening of the general population for obstructive sleep apnoea (OSA).

- Will help trained GDPs keep within Dental Protection Ltd guidelines.

- Encourages a multidisciplinary approach to the management of snoring patients and those suspected of having OSA.

- Will increase the quality and number of referrals to Specialist Respiratory Centres.

\author{
J. Stradling ${ }^{1}$ and R. Dookun ${ }^{2}$
}

VERIFIABLE CPD PAPER

\begin{abstract}
Snoring is not necessarily a benign condition; it can be linked to the serious condition obstructive sleep apnoea (OSA). In some cases mandibular repositioning devices can be an effective treatment for simple snoring and mild to moderate sleep apnoea, and these devices can be provided by dentists (with appropriate training and in line with Dental Protection Ltd guidelines). Until now, the dental profession has not been given any guidance on how to differentiate between patients who may be treated without further reference to medical colleagues (ie simple snorers), and those who should be referred for specialist assessment. The aim of this paper is to facilitate safe treatment of snoring and OSA and to protect dentists by explaining an accepted method for screening patients for obstructive sleep apnoea.
\end{abstract}

\section{BACKGROUND}

\section{Snoring}

Loud and frequent snoring can be more than just a nuisance to sleep partners; it can disrupt whole households and has associated inherent health risks. It is also the primary symptom of OSA, which is a serious medical condition; $30-50 \%$ of asymptomatic snorers will have some degree of sleep apnoea. ${ }^{1-3}$

Snoring is common; almost half of all adults snore at least occasionally and 30\% of adults and $60 \%$ of men over the age of 60 years are habitual snorers. ${ }^{4}$ Snoring is more common in men than in pre-menopausal women which may be due to a greater tendency to male upper body (and neck) fat deposition. After the menopause, female fat distribution becomes more male-like and the prevalence of snoring in women approaches that seen in males.

\footnotetext{
Professor of Respiratory Medicine, Director of the Osle Chest Unit and Respiratory Sleep Unit, Oxford Centre for Respiratory Medicine, Churchill Hospital, Oxford, OX3 9UU; ${ }^{2 *}$ General Practitioner, Cleveland House Dental Practice, La Grande Rue, St Martins, Guernsey, GY4 6LO, Channel Islands

*Correspondence to: Dr Roy Dookun

Email:dookun@guernsey.net
}

\section{Refereed Paper}

Accepted 6 December 2008

DOI: 10.1038/sj.bdj.2009.214

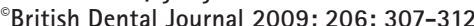

The snoring noise is caused by a decrease in pharyngeal muscle tone during sleep, allowing pharyngeal wall collapse in response to the subatmospheric intra-pharyngeal pressures that develop during inspiration. The turbulent inspiratory air flow in the compliant pharynx causes the noise. Snoring is often worse in people with a crowded pharynx (large tonsils, relative retro or micrognathia, obesity or macroglossia), and when sufferers sleep in the supine position as the antero-posterior dimensions of the pharynx are further decreased due to the effects of gravity.

\section{Sleep apnoea}

Not all snorers have OSA, but virtually all apnoeics snore. Sleep apnoea occurs if the pharyngeal compliance increases sufficiently with sleep onset to allow the muscular pharyngeal tube to narrow critically or collapse completely. Breathing is reduced or stops, oxygen levels fall and carbon dioxide levels rise. Respiratory drive is then increased, partial awakening occurs in response, pharyngeal tone increases again, and breathing restarts.

An apnoeic event is usually described as a cessation of airflow (to $<10 \%$ of previous flow) for $>10$ seconds. A hypopnoea is usually defined as a $>50 \%$ decrease in airflow for $>10$ seconds, usually associated with an oxygen desaturation of $4 \%$ or more. There are two scales which are commonly used to assess the presence and severity of sleep apnoea; the apnoea hypopnoea index (AHI), and the oxygen desaturation index (ODI) (see Table 1). These two scales are very similar, with only a slight difference in the threshold between mild and moderate sleep

\section{Table 1 Classification of the severity of sleep apnoea}

\begin{tabular}{l|l|l} 
AHI & ODI & $\begin{array}{l}\text { Severity of } \\
\text { sleep apnoea }\end{array}$ \\
$\begin{array}{l}\text { Frequency of apnoeas } \\
\text { and hypopnoeas }\end{array}$ & $\begin{array}{l}\text { Frequency of transient reductions } \\
\text { in oxygen saturation of } 4 \% \text { or more }\end{array}$ & No significant \\
\hline$<5 / \mathrm{hr}$ & $<5 / \mathrm{hr}$ & Mild \\
\hline $5-15 / \mathrm{hr}$ & $5-10 / \mathrm{hr}$ & Moderate \\
\hline $16-30 / \mathrm{hr}$ & $11-30 / \mathrm{hr}$ & Severe \\
\hline$>30 / \mathrm{hr}$ & $>30 / \mathrm{hr}$ &
\end{tabular}


apnoea. According to the National Institute for Health and Clinical Excellence technology appraisal, OSA is considered mild when a sleep study records an AHI of 5-14, moderate when the AHI is 15-30 and severe when the AHI is $>30$. In addition to the AHI, the severity of symptoms are also important.

The partial awakenings or cerebral micro arousals of sleep apnoea are associated with sympathetic stimulation and cause large increases in blood pressure, potentially damaging vascular endothelial linings, and may increase insulin resistance. OSA is believed to exacerbate cardiovascular disease (angina and myocardial infarction) and hypertension. It is associated with increased cardiovascular mortality, morning headaches, impotence, nocturnal bruxism, gastro-oesophageal reflux disorder, insulin resistant diabetes and cerebrovascular problems (strokes and transient ischaemic attacks). ${ }^{5}$ However, the exact link between sleep apnoea and cardiovascular damage is still unclear and the associations of OSA with other abnormalities is still disputed. ${ }^{6}$ This is mainly because OSA itself is associated with obesity, particularly central obesity, as are many of the components of the metabolic syndrome which increase vascular morbidity and mortality. When OSA is also associated with excessive daytime sleepiness it is called obstructive sleep apnoea hypopnoea syndrome (OSAHS). Excessive daytime sleepiness, which can be assessed by the Epworth Sleepiness Scale (ESS) (see Table 2), can have serious implications including increased motor vehicle and workrelated accidents, poor job performance, depression, family discord and decreased quality of life..$^{7-9}$ Excessive daytime sleepiness due to undiagnosed sleepdisordered breathing could account for a significant proportion of motor vehicle accidents. ${ }^{9}$

It is estimated that there are over 12 million sufferers of OSA with varying degrees of sleepiness in the USA; it is more common than asthma, and is nearly as common as diabetes. Known risk factors for OSA are crowded upper airway anatomy, endocrine abnormalities (eg untreated hypothyroidism), acromegaly, genetic diseases predisposing to microg

\section{Table 2 The Epworth Sleepiness Scale}

How likely are you to doze off or fall asleep in the situations described below, in contrast to feeling just tired? This refers to your usual way of life in recent times.

Even if you haven't done some of these things recently try to work out how they would have affected you.

Use the following scale to choose the most appropriate number for each situation:

$0=$ would never doze

1 = Slight chance of dozing

2 = Moderate chance of dozing

$3=$ High chance of dozing

\begin{tabular}{|c|c|c|}
\hline \multicolumn{2}{|c|}{ 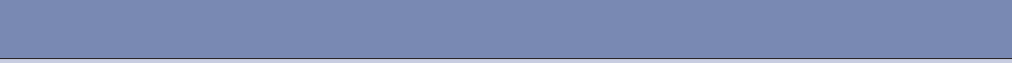 } & Score \\
\hline \multicolumn{2}{|c|}{ Sitting and reading } & \\
\hline \multicolumn{2}{|c|}{ Watching TV } & \\
\hline \multicolumn{2}{|c|}{ Sitting, inactive in a public place (theatre, meeting) } & \\
\hline \multicolumn{2}{|c|}{ As a passenger in a car for an hour without a break } & \\
\hline \multicolumn{2}{|c|}{ Lying down to rest in the afternoon when circumstances permit } & \\
\hline \multicolumn{2}{|c|}{ Sitting and talking to someone } & \\
\hline \multicolumn{2}{|c|}{ Sitting quietly after a lunch without alcohol } & \\
\hline \multicolumn{2}{|c|}{ In a car, whilst stopped for a few minutes in traffic } & \\
\hline \multicolumn{2}{|c|}{ Total/24 } & \\
\hline \multicolumn{3}{|c|}{ Epworth Sleepiness Scale interpretation and advice to patients } \\
\hline \multicolumn{3}{|l|}{ Score } \\
\hline $0-7$ & Well within the normal range. 'It is unlikely that you are abnormally sleepy' & \\
\hline $8-9$ & Top end of normal range. 'You have an average amount of daytime sleepiness' & \\
\hline $10-15$ & $\begin{array}{l}\text { Above the conventional top end of normal although many normal people will ha } \\
\text { of sleepiness for lifestyle reasons. 'You may be excessively sleepy depending on } \\
\text { You may want to consider seeking medical advice' }\end{array}$ & $\begin{array}{l}\text { this level } \\
\text { e situation. }\end{array}$ \\
\hline $16-24$ & Clearly abnormal. 'You are excessively sleepy and should consider seeking medic & attention' \\
\hline
\end{tabular}

\section{Table 3 Medical history questionnaire}

Relevant medical issues requiring prior consideration because:

- of their association with OSA

- the condition may be exacerbated by OSA

- it may limit the use of an intra oral appliance.

However, they are not in themselves absolute contraindications to treatment.

\begin{tabular}{|c|c|c|}
\hline Cardiac & Respiratory & Cerebral \\
\hline $\begin{array}{l}\text { Angina/Chest pain } \\
\text { Hypertension } \\
\text { Previous myocardial } \\
\text { infarction (MI) } \\
\text { Palpitations } \\
\text { Oedema }\end{array}$ & $\begin{array}{l}\text { Shortness of breath } \\
\text { Asthma (if not well controlled) } \\
\text { Regular cough } \\
\text { Chronic obstructive airways } \\
\text { disease (COAD) }\end{array}$ & $\begin{array}{l}\text { Previous cerebro vascular } \\
\text { incident (CVI) } \\
\text { Transient ischaemic attacks (TIAs) }\end{array}$ \\
\hline General & Recent onset snoring & Evidence of upper airway resistance \\
\hline $\begin{array}{l}\text { Epilepsy - IF WELL CONTROLLED } \\
\text { Diabetes if poorly controlled } \\
\text { or insulin resistant } \\
\text { Hypothyroidism } \\
\text { Gastro oesophageal } \\
\text { reflux disease (GORD) } \\
\text { Menopause }\end{array}$ & $\begin{array}{l}\text { Recent onset snoring may } \\
\text { indicate new onset hypothy- } \\
\text { roidism, sudden weight gain, } \\
\text { development of a pharyngeal } \\
\text { space occupying lesion, } \\
\text { menopause }\end{array}$ & $\begin{array}{l}\text { Small throat - pharyngeal wall } \\
\text { behind soft palate not visible } \\
\text { Large tongue } \\
\text { Large uvula } \\
\text { Large tonsils } \\
\text { Nasal obstruction } \\
\text { Previous surgery for snoring } \\
\text { and/or OSA } \\
\text { Noisy breathing whilst awake }\end{array}$ \\
\hline
\end{tabular}




\begin{tabular}{|c|c|c|c|c|}
\hline \multicolumn{5}{|c|}{ Table 4 Patient assessment } \\
\hline \multicolumn{5}{|c|}{ Patient } \\
\hline$E S S>10$ & \multicolumn{2}{|l|}{ (Table 2) } & NO & YES \\
\hline \multirow[t]{2}{*}{ OSA history } & \multicolumn{2}{|c|}{$\begin{array}{l}\text { Presence of any ONE of these major symptoms: } \\
\text { - Witnessed apnoeas } \\
\text { - Choking during sleep } \\
\text { - Obesity (BMI >30, neck circum }>17 " / 43 \mathrm{~cm} \text { ) }\end{array}$} & NO & YES \\
\hline & \multicolumn{2}{|c|}{$\begin{array}{l}\text { Presence of any TWO OR MORE of these minor symptoms: } \\
\text { - Patients hearing the end of their 'own snore' } \\
\text { - Patients having to sleep sitting up } \\
\text { - Nocturia }\end{array}$} & NO & YES \\
\hline Uncontrolled epilepsy & & & NO & YES \\
\hline \multicolumn{3}{|c|}{$\begin{array}{l}\text { GMP referral to be considered? (a 'YES' response to any section may indicate the need } \\
\text { for patient referral to GMP for further investigation and diagnosis) }\end{array}$} & NO & YES \\
\hline \multicolumn{5}{|c|}{$\begin{array}{l}\text { Snoring/OSA predisposing factors to be addressed first } \\
\text { These factors must be assessed and may provide a therapeutic alternative or adjunct to treatment }\end{array}$} \\
\hline \multicolumn{5}{|c|}{$\begin{array}{l}\text { Smoking } \\
\text { Evening alcohol } \\
\text { Supine only snoring } \\
\text { Use of sedative medication } \\
\text { Weight }\end{array}$} \\
\hline \multicolumn{5}{|c|}{ Medical history must be assessed and taken into consideration (Table 3) } \\
\hline \multicolumn{5}{|c|}{$\begin{array}{l}\text { Appliance assessment factors must be assessed and may influence your decision to provide an } \\
\text { intra oral device: }\end{array}$} \\
\hline \multicolumn{2}{|c|}{ Migraine, chronic headaches } & \multicolumn{3}{|l|}{ Pain in or around TMJ } \\
\hline \multicolumn{2}{|c|}{ Limited mandibular opening/protrusion } & \multicolumn{3}{|c|}{ Skeletal/craniofacial considerations } \\
\hline \multicolumn{2}{|l|}{ Periodontal status } & \multicolumn{3}{|l|}{ Pronounced gag reflex } \\
\hline \multicolumn{2}{|c|}{ Mandibular locking/TMJ dysfunction } & \multicolumn{3}{|c|}{ Sleep parafunction - bruxism, clenching } \\
\hline \multicolumn{2}{|l|}{ Ear pain/stuffiness } & \multicolumn{3}{|l|}{ Dental status } \\
\hline \multicolumn{5}{|c|}{ 2) Patient assessment algorithm } \\
\hline \multicolumn{5}{|c|}{$\begin{array}{l}\text { - }<10 \text {, go to algorithm (Fig. 1) } \\
\text { If there are no OSA pointers (negative } 0 \\
\text { predisposing factors and appliance fact } \\
\text { algorithm (LEFT hand pathway). If there } \\
\text { overnight monitoring (RIGHT hand path } \\
\text { - } 10-15 \text {, go to algorithm (Fig. 2) } \\
\text { - }>15 \text {, go to algorithm (Fig. 3) which indic }\end{array}$} \\
\hline
\end{tabular}

nathia (Pierre Robin Syndrome), alcohol or sedative use (especially if taken late in the evening), smoking, being male and being overweight (especially around the neck)..$^{10}$ OSA progressively worsens with age. ${ }^{11,12}$

Lifestyle changes, such as avoiding sedative medication and alcohol close to bedtime, regular exercise, losing weight, and sleeping on one's side can help to reduce snoring and OSA. Available therapies which have been well documented include mandibular repositioning devices (MRDs) and continuous positive airway pressure (CPAP). ${ }^{13,14}$ Further research is required on the efficacy of surgery, including experimental techniques such as soft palate implants and hypogossal nerve stimulation. ${ }^{15}$

\section{Treatment of snoring and OSA by GDPs}

Dentists are in a position to provide primary treatment for snoring and mild OSA, as well as second-line treatment for more severe OSA if first line CPAP treatment fails due to lack of compliance. Unlike GMPs, dentists can assess the suitability of patients for treatment with an MRD and can arrange adequate follow up treatment to monitor the effects of such devices on the teeth, periodontal tissues and the temporomandibular joints (TMJs). MRDs are well tolerated and effective in the treatment of snoring, sleep quality is enhanced and significant reductions in ESS are recorded in a high percentage of patients. They can, however, produce unwanted side effects, both temporary and long term. The most commonly reported short term side effects are jaw discomfort, tooth tenderness, excessive salivation or dry mouth. Dental study model measurements however have shown reductions in overbite and overjet with long term MRD use although these changes were usually clinically insignificant, the findings have been supported in cephalometric analysis. Changes are usually limited to the mandibular incisors and molars moving anteriorly with no significant changes recorded in the maxilla. ${ }^{16}$ For this reason, it would be considered mandatory that accurate, orthodontically trimmed preoperative study casts should be prepared and a recall evaluation programme instituted to monitor the effects on the periodontium, teeth, TMJ and occlusion if treatment with an oral device is to be considered.

However, such anti-snoring devices could be counter-productive in patients with undiagnosed OSA, masking symptoms and resulting in late diagnosis of the condition. In a position statement on snoring and OSA released in 2005 by the largest defence organisation, Dental Protection Ltd, it is stipulated that where OSA is present, anti-snoring devices should only be provided as part of an integrated treatment plan, where the GDP is working in conjunction with a specialist ENT or Respiratory physician. ${ }^{17}$ Correct diagnosis of simple snoring and OSA is therefore vital to ensure that patients receive appropriate treatment.

\section{Screening for OSA}

The regular contact that dentists have with patients, their regular review of medical histories and examination of the soft tissues, teeth, periodontal health and the condition of the TMJs, means that dentists are ideally placed to screen for OSA, assess the suitability of the patient for treatment and provide an intra oral 
device where indicated. Because patients who snore may present to their GDPs before their GMPs, GDPs have the potential to recognise patients with OSA who might not otherwise be diagnosed. However, Dental Protection Ltd stipulate that patients who exhibit signs or symptoms of OSA must be referred for medical assessment. ${ }^{17}$ It is therefore imperative that, prior to the provision of any antisnoring device, patients are given a thorough and documented assessment to identify signs and symptoms of OSA. Appropriate training is crucial. Indeed, Dental Protection stipulates that in order to apply for assistance, dentists must have undergone a documented training course in the provision of these appliances, including training in the appropriate screening for OSA.

Until now, the dental profession has not been given any guidelines on how to differentiate between patients who may be treated without referral to medical colleagues, and those who should be referred for specialist assessment.

Unlike the USA, in Great Britain, specialist medical assessment of snorers for the diagnosis of OSA cannot yet be routinely and quickly carried out with, for example, overnight polysomnography, due to both availability and financial constraints. Indeed, some sleep physicians would consider this level of screening as excessive, preferring simpler alternatives. If between $25-35 \%$ of the adult population snore and between $30-50 \%$ of snorers suffer from OSA, already busy sleep units would be unlikely to welcome a rash of referrals just so that dentists could treat simple snorers.

\section{DEVELOPMENT OF A SCREENING PROTOCOL}

In order to try to overcome the problems that dentists face in treating snoring patients and in screening for OSA/ OSAHS, a Sleep Medicine Working Party was set up in cooperation with the British Society for Dental Sleep Medicine (BSDSM), comprising leading respiratory physicians and GDPs to develop a pre-treatment screening protocol for snoring patients. The intellectual, practical and evidence base reasoning behind this protocol is not explained

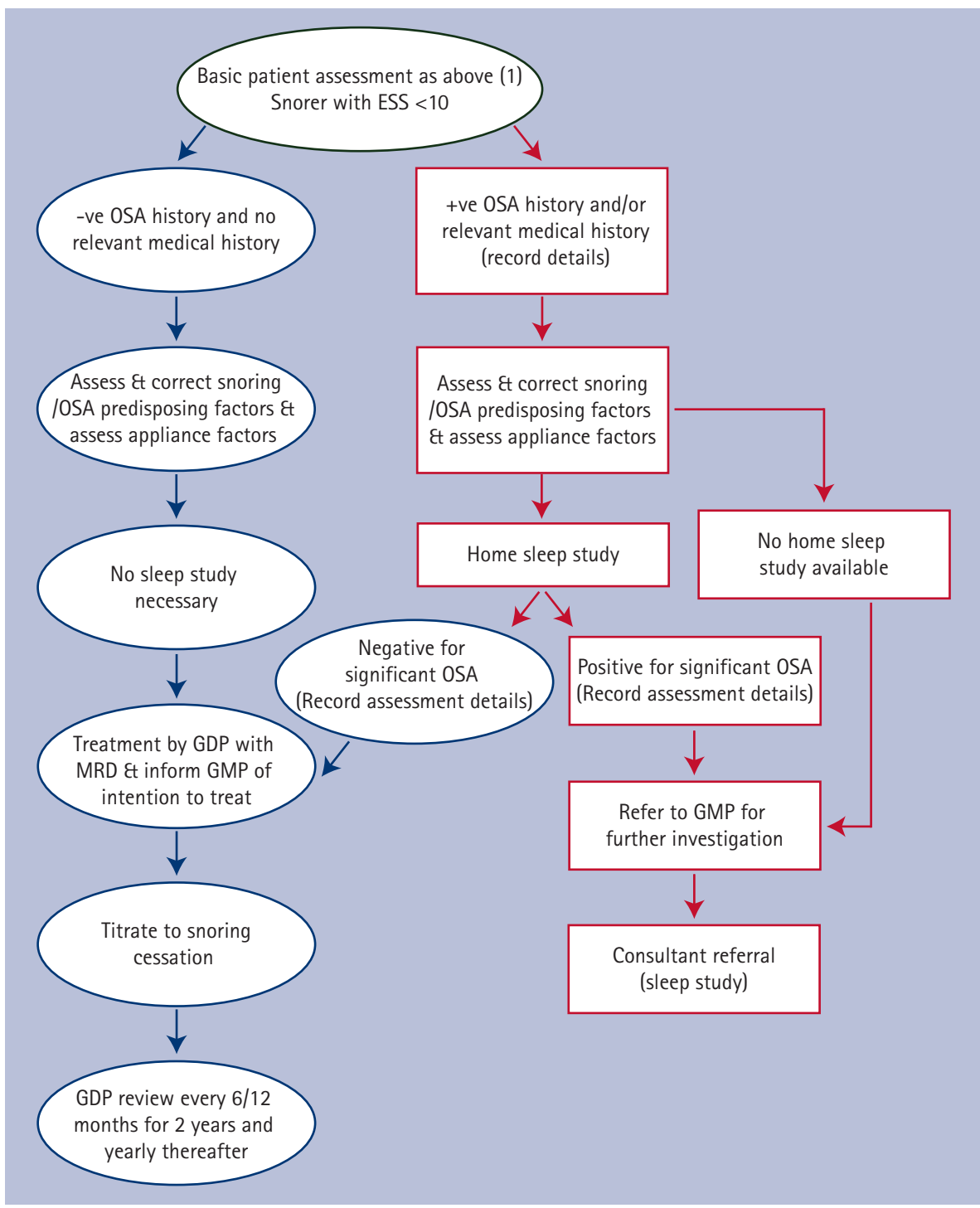

Fig. 1 Patient assessment algorithm

here, and it is strongly recommended that practitioners attend training where the protocol is discussed fully. According to the protocol, patients should be carefully assessed using the ESS (Table 2), a medical history questionnaire (Table 3), and a basic patient assessment (see Table 4). The appropriate treatment/ referral path can be determined from an algorithm created by the working party, the basic premise of which is presented in Figure 1 - left hand pathway. The aim of the protocol is to prevent dentists from inadvertently providing snoring appliances for patients with OSA or OSAHS, thus denying them the opportunity for more appropriate treatment. If the patient is carefully assessed, and the algorithm followed, it is unlikely that serious OSA or OSAHS will be missed and, in the unlikely event the GDP's judgement was questioned, it could be argued that the practitioner applied a standard of care supported by a consensus view of a responsible body of opinion. The BSDSM pre-treatment screening protocol has been accepted by Dental Protection Ltd and was presented to the British Thoracic Society Sleep Advisory Group in Feb 2007 who accepted the principles and value of such an approach.

With respect to the treatment of snoring and OSA, it is envisaged that two distinctive types of general dental practice will evolve. Some practitioners will use the paper screening protocol (Table 4) and only choose to treat non-obese patients (obesity defined as having a neck circumference of $17 \% / 43 \mathrm{~cm}$ or above and/or a Body Mass Index (BMI) $>30$. The BMI is calculated by dividing the patients weight in kilograms by their height in metres squared), non-somno- 
lent snorers with an ESS $<10$ and no relevant OSA/medical history.

The second, more experienced (and suitably trained) practitioner would provide ambulatory screening using the advanced protocol (Figs 1-3) for both those patients with an ESS of $\leq 10$ and a positive OSA/medical history, and those with a moderate ESS (10-15). It is important to note that while an ESS $>10$ may indicate OSAHS, it may also be due to lifestyle issues such as shift work, too little sleep time, sleep disturbance from babies, or a noisy environment. This second group of practitioners would take responsibility for interpreting the results of the ambulatory screening, assessing the likelihood of sleep apnoea being present and making the decision whether to provide an MRD or refer the patient for specialist diagnosis. GDPs would need to justify the effectiveness of their chosen ambulatory screening device according to current recommended practice.

\section{Home overnight monitoring}

A further interesting development in the screening of OSA is the use of home overnight recording screening devices. There are a number of devices on the market ranging from inexpensive twin channel oximeters (recording overnight oxygen saturations and pulse rate) to more sophisticated multi-channel monitors that measure chest and/or abdominal movement, nasal flow, oximetry, pulse rate, snoring and body position.

New guidelines drawn up by the Sleep Medicine Working Party provide that suitably trained dentists may use monitoring devices such as ambulatory recording pulse oximeters to screen for the presence of sleep breathing disorders. These monitors can also be used to verify the effectiveness of an oral device when used to treat OSA. From a dento-legal point of view, dentists who use these monitors should be able to demonstrate that they have sufficient training in the use and interpretation of these devices.

A very clear distinction should be made at this point. Appropriately trained dentists may use these devices to screen for the presence of sleep related breathing disorders. They may not diagnose

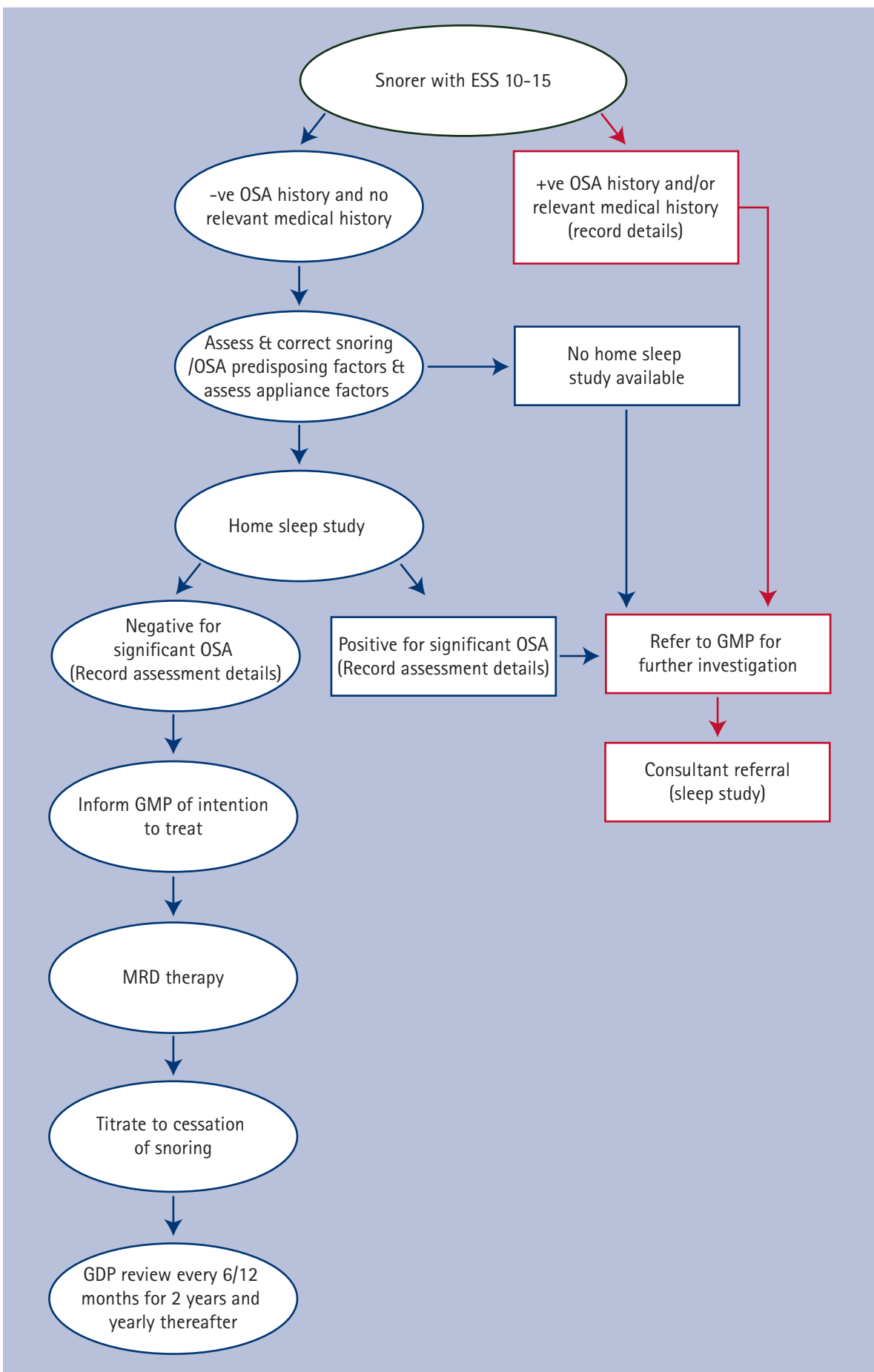

Fig 2. The advanced protocol for ambulatory screening

although they may suspect OSA, and they may not treat OSA without prescription from an appropriate physician. Use of the screening protocol and a home overnight monitoring device will enable GDPs to confidently screen out and treat simple snorers, and provide the greatest possible service to the many serious OSA sufferers who will be identified by the screening and fast tracked for formal diagnosis and treatment.

When in doubt the advice would always be to refer. The development of a professional relationship with a local sleep physician would be ideal as an opinion could be sought on borderline tracings.

\section{CONCLUSION}

GDPs have a clear dual role in assessing snorers presenting for advice. Firstly, they are in a prime position to identify patients with OSA and OSAHS, and refer them on to the right service. Secondly, they can provide effective devices for the control of snoring and mild OSA, 


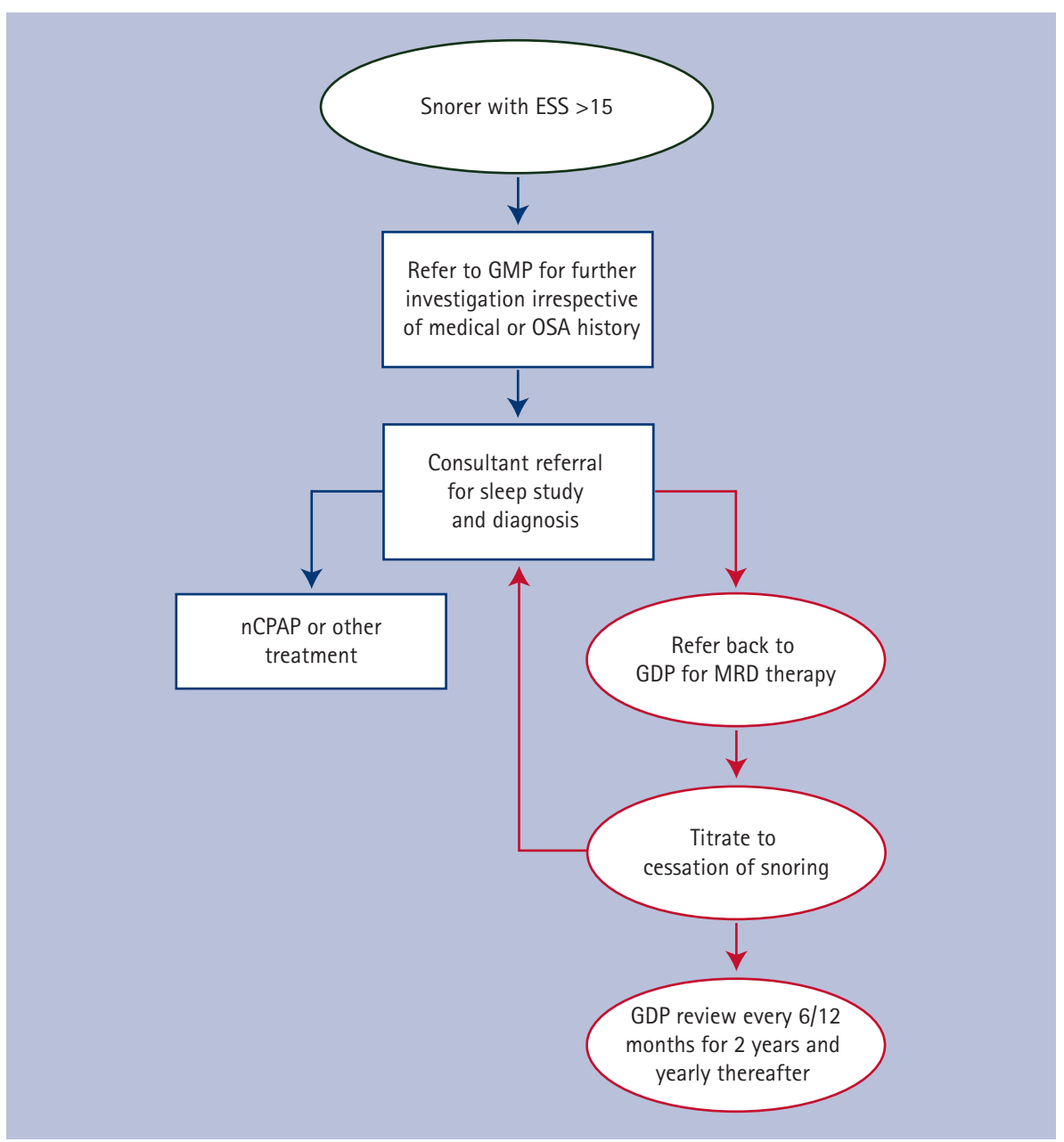

Fig. 3 Patient assessment algorithm

as well as for more severe OSA as second line therapy. However, such a service needs to be provided with adequate prior training and a consistent assessment tool. The protocol is offered as a draft, and no doubt will evolve as more
3. Lindberg E, Janson C, Svardsudd K, Gislason T et al. Increased mortality among sleepy snorers: a prospective population based study. Thorax 1998; 53: 631-637.

4. Olson L G, King M T, Hensley M J, Saunders N A. A community study of snoring and sleep-disordered breathing. Prevalence Am J Respir Crit Care Med 1995: 152: 711-716.

5. Robinson G V, Stradling J R, Davies R J. Sleep. 6: obstructive sleep apnoea/hypopnoea syndrome and hypertension. Thorax 2004: 59: 1089-1094.

6. Marin J M, Carrizo S J, Vicente E, Agusti A G. Long-term cardiovascular outcomes in men with obstructive sleep apnoea-hypopnoea with or without treatment with continuous positive airway pressure: an observational study. Lancet 2005; 365: 1046-1053.

7. Johns M W. A new method for measuring daytime sleepiness: the Epworth sleepiness scale. Sleep 1991; 14: 540-545.

8. George C F. Reduction in motor vehicle collisions following treatment of sleep apnoea with nasal CPAP. Thorax 2001; 56: 7-12.

9. Young T, Blustein J, Finn L, Palta M. Sleep-disordered breathing and motor vehicle accidents in a population-based sample of employed adults. Sleep 1997; 20: 608-613

10. Young T, Palta M, Dempsey J, Skatrud J et al. The occurrence of sleep-disordered breathing among middle-aged adults. N Eng/ J Med 1993; 328: 1230-1235.

11. Stradling J R, Crosby J H. Predictors and prevalence of obstructive sleep apnoea and snoring in 1001 middle aged men. Thorax 1991; 46: 85-90.

12. Davies R J, Stradling J R. The relationship between neck circumference, radiographic pharyngeal anatomy, and the obstructive sleep apnoea syndrome. Eur Respir J 1990; 3: 509-514.

13. Ferguson $K A$, Cartwright $R$, Rogers $R$, SchmidtNowara W. Oral appliances for snoring and obstructive sleep apnea: a review. Sleep 2006; 29: 244-262.

14. White J, Cates C, Wright J. Continuous positive airways pressure for obstructive sleep apnoea. Cochrane Database Syst Rev 2006; 19: CD001106. Review.

15. Bridgman S A, Dunn K M. Surgery for obstructive sleep apnoea Cochrane Database Syst Rev 2000; 2: CD001004. Review.

16. Hammond R J, Gotsopoulos H, Shen G, Petocz $P$ et al. A follow-up study of dental and skeletal changes associated with mandibular advancement splint use in obstructive sleep apnea. Am J Orthod Dentofacial Orthop 2007; 132: 806-814.

17. http://www.dentalprotection.org/united_kingdom/news and information/position statements/20041001_ps_sleepapnoea.aspx 